\title{
A CLASS OF ORTHOGONAL POLYNOMIALS OF A NEW TYPE
}

\author{
M. DUTTA and \\ KANCHAN PRABHA MANOCHA \\ S.N. Bose Institute of Physical Sciences \\ University of Calcutta \\ 92, Acharya Prafulla Chandra Road \\ Calcut ta-700009, INDIA \\ (Received June 19, 1982)
}

\begin{abstract}
The purpose of this paper is to study a class of polynomials $F_{n}^{m}(x, \lambda, \nu)$ associated with a class of $\mathrm{m}$ differential equations, where $\lambda$ is fixed, $\mathrm{n}$ is a variable parameter, $\mathrm{m}$ is fixed positive integer and $\nu$ is a non-negative integer $<\mathrm{m}$. We also obtain all the important properties of this class of polynomials including Rodrigues' formulas, generating function, and recurrence relations. Several special cases of interest are obtained from our analysis.
\end{abstract}

KEY WORDS AND PHRASES. Function space, weight function, generating function, and recurrence relation. 1980 MATHEMATICS SUBJECT CLASSIFICATION CODES. 33A65, 34A30.

1. INTRODUCTION.

The classical orthogonal polynomials, including Jacobi, Gegenbauer, Legendre, Tchebicheff, Laguerre and Hermite polynomials and some of their generalizations, have been studied in great details (Erdélyi et al [1]). Each of these classes of polynomials satisfies one differential equation, one Rodrigues' formula and a pure recurrence relation.

In 1965, Dutta and lfore [2] obtained the generalization of Legendre polynomials with the help of Schmidt-orthonormalization process, in this it is seen that this class of polynomials satisfies two different differential equations, two Rodrigues' formulas, etc., for even and odd values of the variable parameter in the same function space. 
Similar generalization of the class of Hermite polynomials, so that two differential equations, two Rodrigues' formulas and two recurrence relations for even and odd values of the variable parameter and associated in the same function space, was obtained, and studied in some details by Dutta, Chatterjea, Ghosh [3] and Dutta, Chatterjea and More [4]. Earlier in the paper of Krall and Frink [5], some similar result i.e. two different differential equations in the discussion of a class of functions written by suitable modification of Bessel functions are seen, but this pecularity is not studied with due emphasis.

Theoretical justification of the association of a pair of differential equations with a single class of orthogonal functions has been discussed in some details in Dutta and Bhattacharya [6]. In a subsequent paper of Dutta [7], the possibility of a class of orthogonal polynomials having m number of differential equations, m recurrence relations, etc. ( $m$ being a fixed natural number) is established.

In this paper following the suggestions of Dutta [7], it is shown how and when solutions of m differential equations may belong to the same class of orthogonal polynomials. This new class of orthogonal polynomials satisfies m Rodrigues' formulas, m recurrence relations, etc., where m being the number of partitions of the variable parameter $\mathrm{n}$.

Let us consider a class of m differential equations:

$$
y^{\prime \prime}-\left(m x^{m-1}-\frac{\lambda}{x}\right) y^{\prime}+\left(m x^{m-2}-\frac{v(\lambda+v-1)}{x^{2}}\right) y=0
$$

where $\lambda$ is fixed, $\mathrm{n}$ is a variable parameter, $\mathrm{m}$ is a fixed positive integer where $\nu$ is a non-negative integer $<\mathrm{m}$.

Here we see that for different values of $v$ we shall obtain $m$ different differential equations, but solutions of these differential equations are orthogonal in the same function space $L_{2}^{W(x, \lambda)}(I)$ where

$$
\begin{aligned}
& \qquad \mathrm{w}(\mathrm{x}, \lambda)=e^{-\mathrm{x}^{\mathrm{m}}}|\mathrm{x}|^{\lambda+\mathrm{m}-2}, \\
& \mathrm{I}=(0, \infty) \text { when } \mathrm{m} \text { is odd, } \\
& =(-\infty, \infty) \text { when } \mathrm{m} \text { is even. }
\end{aligned}
$$

of course for an odd $m$, as $I$ is $(0, \infty)$ so $|x|$ is obviously replaced by $x$. The intervals are taken from the consideration of convergence of the integral defining 
or thogonality.

2. THE CLASS OF POLYNOMIALS $\left\{\mathrm{F}_{\mathrm{n}}^{\mathrm{m}}(\mathrm{x}, \lambda, \nu)\right\}$ AND THEIR ORTHOGONALity.

Solving the differential equation (1.1), we obtain a class of functions

$$
F_{n}^{m}(x, \lambda, \nu)=x^{\nu} \sum_{k=0}^{\left[\frac{n-\nu}{m}\right]} \frac{\left(-\frac{n-v}{m}\right)_{k}\left(x^{m}\right)^{k}}{\left(\frac{2 v+\lambda+m-1}{m}\right)_{k} k !}
$$

for running parameter $\mathrm{n}$ and for fixed $\lambda$, $\mathrm{m}$ the functions will reduce to polynomials for $\mathrm{n} \equiv \nu(\bmod \mathrm{m})$ and $\nu=0,1, \ldots, \mathrm{m}-1$, where $\nu$ is a non-negative integer $<\mathrm{m}$. Now all the polynomials for different values of $n$ with $n \equiv \nu$ (mod $m$ ) form a class of orthogonal polynomials.

For differnet values of $v$ corresponding to a particular (fixed) value of $\mathrm{m}$ we shall obtain m different classes of polynomials.

ORTHOGONALITY. Following the technique of Dutta and Bhattacharya [6] and Dutta [7], we can calculate the weight function $w(x, \lambda)=e^{-x^{m}}|x|^{\lambda+m-2}$ of the function space properly associated with the class of differential equations and establishes the orthogonality relation:

$$
\int_{I} e^{-x^{m}}|x|^{\lambda+m-2} F_{\mu_{i}}^{m}(x, \lambda, \nu) F_{\mu_{j}^{\prime}}^{m}(x, \lambda, \nu) d x=\delta_{\mu_{i} \mu_{j}^{\prime}}
$$

where

$$
\begin{aligned}
I & =(0, \infty) \text { if } \mathrm{m} \text { is odd, } \\
& =(-\infty, \infty) \text { if } \mathrm{m} \text { is even. }
\end{aligned}
$$

3. THE POLYNOMIALS AS ${ }_{2} \mathrm{~F}_{0}$.

From the equation (2.1) by reversing the order of summation, we can write at once

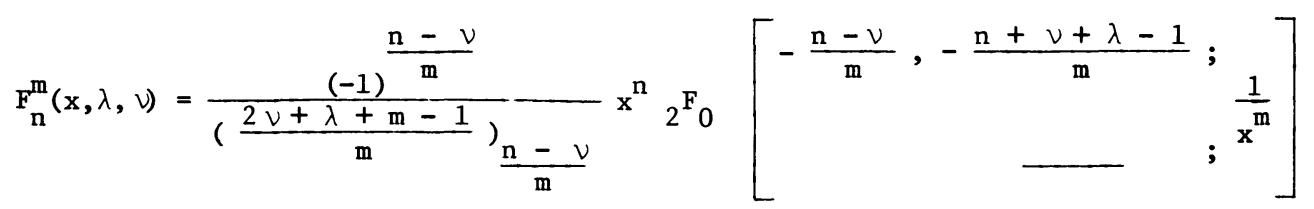

\section{RELATION WITH LAGUERRE POLYNOMIALS.}

In (2.1) replacing $x$ by $\sqrt[m]{x}$ (here we are taking the real positive root of 


$$
\begin{aligned}
& F_{m+}^{m}(\sqrt[m]{x}, \lambda, v)=x^{\frac{v}{m}} \sum_{k=0}^{n} \frac{(-n)_{k} x^{k}}{\left(\frac{2 v+\lambda+m-1}{m}\right)_{k} k !} \\
& =x_{1}^{\frac{\nu}{m}} F_{1}\left[\begin{array}{cc}
-n ; & x \\
\frac{2 \nu+\lambda+m-1}{m} ; &
\end{array}\right]
\end{aligned}
$$

where $\mathrm{n} \equiv \nu(\bmod \mathrm{m})$.

This can be written in terms of Laguerre polynomials:

$$
\mathrm{F}_{\mathrm{mn}+}^{\mathrm{m}}(\sqrt[m]{\mathrm{x}}, \lambda, v)=\frac{\frac{v}{\mathrm{~m}} \mathrm{n} !}{\left(\frac{2 v+\lambda+m-1}{m}\right)_{n}} \mathrm{~L}_{\mathrm{n}}^{\left(\frac{2 v+\lambda-1}{m}\right)}(\mathrm{x})
$$

5. RODRIGUES' FORMULAS.

For Laguerre polynomials, Rodrigues' formula is given by

$$
L_{n}^{(\alpha)}(x)=\frac{x^{-\alpha} e^{x}}{n !} D^{n}\left(e^{-x} x^{n+\alpha}\right) \text { where } D \equiv \frac{d}{d x} \text {. }
$$

So, from (4.2), we get

$$
F_{m n+\nu}^{m}(\sqrt[m]{x}, \lambda, \nu)=\frac{\frac{\nu}{m} n !}{\left(\frac{2 \nu+\lambda+m-1}{m}\right)_{n}} \times \frac{\frac{1-\lambda-2 \nu}{m} e^{x}}{n !} D^{n}\left(e^{-x} x^{n+\frac{2 \nu+\lambda-1}{m}}\right)
$$

or

$$
F_{m n+\nu}^{m}(\sqrt[m]{x}, \lambda, \nu)=\frac{\frac{1-\lambda-\nu}{m}}{\left(\frac{2 v+\lambda+m-1}{m}\right)_{n}} D^{n}\left(e^{-x} x^{n+\frac{2 \nu+\lambda-1}{m}}\right)
$$

Obviously, for different values of $\nu(\nu=0,1,2, \ldots, m-1)$, we shall obtain $m$ different Rodrigues' formulas.

6. GENERATING FUNCTIONS.

Equation (2.1) can be written as:

$$
\text { - } \mathrm{F}_{\mathrm{m} n+v}^{\mathrm{m}}(\mathrm{x}, \lambda, \nu)=\mathrm{x}^{\nu} \sum_{\mathrm{k}=0}^{\mathrm{n}} \frac{(-\mathrm{n})_{k}\left(\mathrm{x}^{\mathrm{m}}\right)^{\mathrm{k}}}{\left(\frac{2 v+\lambda+\mathrm{m}-1}{\mathrm{~m}}\right)_{\mathrm{k}} \mathrm{k!}}
$$

Multiplying both sides by $\frac{t^{n}}{n !}$ and taking the sum from $n=0$ to $\infty$,

$$
\sum_{n=0}^{\infty} F_{m n+v}^{m}(x, \lambda, v) \frac{t^{n}}{n !}=\sum_{n=0}^{\infty} x^{v} \sum_{k=0}^{n} \frac{(-n)_{k}\left(x^{m}\right)^{k}}{\left(\frac{2 v+\lambda+m-1}{m}\right)_{k} k !} \frac{t^{n}}{n !}
$$




$$
\begin{aligned}
& =x \sum_{n=0}^{\infty} \frac{t^{n}}{n !} \sum_{k=0}^{n} \frac{(-1)^{k} n ! x^{m k}}{m-k) !\left(\frac{2 v+\lambda+m-1}{m}\right)_{k} k !} \\
& =x^{v} \sum_{n=0}^{\infty} \sum_{k=0}^{\infty} \frac{(-1)^{k} x^{m k} t^{n+k}}{n !\left(\frac{2 v+\lambda+m-1}{m}\right)_{k} k !} \\
& \left.=x^{v} \sum_{n=0}^{\infty} \frac{t^{n}}{n !} \sum_{k=0}^{\infty} \frac{(-1)^{k} x^{m k} t^{k}}{m} \frac{2 v+\lambda+1}{m}\right)_{k} k !
\end{aligned}
$$

Thus the generating function for $\mathrm{F}_{\mathrm{n}}^{\mathrm{m}}(\mathrm{x}, \lambda, \nu)$ is given by the relation

$$
\sum_{n=0}^{\infty} F_{m+\nu}^{m}(x, \lambda, \nu) \frac{t^{n}}{n !}=x^{\nu} e^{t}{ }_{0} F_{1}\left(-; \frac{2 \nu+\lambda+m-1}{m} ;-x^{m} t\right)
$$

To find out the other generating function multiplying both sides of the equation (6.1) by (c) $\frac{t^{n}}{n !}$ and taking the sum from 0 to $\infty$,

$$
\begin{aligned}
& \sum_{n=0}^{\infty}(c)_{n} F_{m n+\nu}^{m}(x, \lambda, \nu) \frac{t^{n}}{n !}=\sum_{n=0}^{\infty} x^{\nu}(c)_{n} \sum_{k=0}^{n} \frac{(-n)_{k} x^{m k}}{\left(\frac{2 v+\lambda+m-1}{m}\right)_{k} k !} \frac{t^{n}}{n !} \\
& =x v \sum_{n=0}^{\infty} \sum_{k=0}^{n} \frac{(c)_{n}(-1)^{k} x^{m k}}{(n-k) !\left(\frac{2 v+\lambda+m-1}{m}\right)_{k} k !} t^{n} \\
& =x^{\nu} \sum_{n, k=0}^{\infty} \frac{(c)_{n+k}(-1)^{k} x^{m k}}{n !\left(\frac{2 v+\lambda+m-1}{m}\right)_{k} k !} t^{n+k} \\
& =x^{\nu} \sum_{k=0}^{\infty} \sum_{n=0}^{\infty} \frac{(c+k)_{n} t^{n}}{n !} \frac{(c)_{k}(-1)^{k} x^{m k} t^{k}}{\left(\frac{2 \nu+\lambda+m-1}{m}\right)_{k} k !} \\
& =x \nu \sum_{k=0}^{\infty} \frac{(c)_{k}(-1)^{k} x^{m k} t^{k}}{\left(\frac{2 \nu+\lambda+m-1}{m}\right)_{k} k !(1-t)^{c+k}} \\
& =(1-t)^{-c} \times \sum_{k=0}^{\infty} \frac{(c)_{k}\left(-x^{m} t\right)^{k}}{\left(\frac{2 \nu+\lambda+m-1}{m}\right)_{k}(1-t)^{k} k !},(6.3)
\end{aligned}
$$

With the choice $c=\frac{2 \nu+\lambda+m-1}{m}$, equation (6.3) can be written as:

$$
\sum_{n=0}^{\infty}\left(\frac{2 v+\lambda+m-1}{m}\right)_{n} F_{m+\nu}^{m}(x, \lambda, \nu) \frac{t^{n}}{n !}=(1-t)^{-\frac{2 v+\lambda+m-1}{m}} x_{0}^{\nu} F_{0}\left(-;-; \frac{-x^{m} t}{(1-t)}\right)
$$

Hence our required generating function is given by 


$$
\sum_{n=0}^{\infty}\left(\frac{2 v+\lambda+m-1}{m}\right)_{n} F_{m n+v}^{m}(x, \lambda, v) \frac{t^{n}}{n !}=x^{v}(1-t)^{-\frac{2 v+\lambda+m-1}{m}} \exp \left(\frac{-x^{m} t}{1-t}\right)
$$

7. RECURRENCE RELATIONS.

In this section, we obtain pure and mixed recurrence relations for the polynomials $\mathrm{F}_{\mathrm{n}}^{\mathrm{m}}(\mathrm{x}, \lambda, v)$.

7.1. DIFFERENTIAL RECURRENCE RELATIONS. In the last section, we have obtained the generating function for the polynomials $F_{n}^{m}(x, \lambda, v)$ given by $(6.2)$ :

$$
\sum_{n=0}^{\infty} F_{m n+v}^{m}(x, \lambda, v) \frac{t^{n}}{n !}=x^{v} e^{t}{ }_{0} F_{1}\left[\begin{array}{cc}
- & -x^{m} t \\
\frac{2 v+\lambda+m-1}{m} & ;
\end{array}\right] \text {. }
$$

So,

$$
\sum_{n=0}^{\infty} x^{-v} F_{m n+v}^{m}(x, \lambda, v) \frac{t^{n}}{n !}=e^{t}{ }_{0} F_{1}\left[\begin{array}{c}
- \\
\frac{2 v+\lambda+m-1}{m}
\end{array}\right]
$$

Let

$$
G(x, t)=e^{t} F_{1}\left(-; \frac{2 \nu+\lambda+m-1}{m} ;-x^{m} t\right)
$$

Differentiating (7.2) partially with respect to $x$ and $t$ and writing $G$ in place of $G(x, t)$, we get

$$
\frac{\partial G}{\partial x}=-m x^{\mathrm{In}-1} t e^{t}{ }_{0} F_{1}^{\prime}\left(-; \frac{2 v+\lambda+m-1}{m} ;-x^{m} t\right)
$$

and

$\frac{\partial G}{\partial t}=e^{t}{ }_{0} F_{1}\left(-; \frac{2 \nu+\lambda+m-1}{m} ;-x^{m} t\right)-x^{m} e_{0}^{t} F_{1}^{\prime}\left(-; \frac{2 \nu+\lambda+m-1}{m} ;-x^{m} t\right),(7.4)$

where prime denotes the differentiation with respect to the argument.

Eliminating ${ }_{0} F_{1},{ }_{0} F_{1}^{\prime}$ from $(7.2),(7,3)$ and $(7.4)$, we see that $G(x, t)$ satisfies the following partial differential equation:

$$
x \frac{\partial G}{\partial x}-m t \frac{\partial G}{\partial t}=-m t G
$$

Substituting G from (7.1)

$$
\begin{array}{r}
x \sum_{n=0}^{\infty}\left[-v x^{-v-1} F_{m+v}^{m}(x, \lambda, v)+x^{-\nu} F_{m n+v}^{m}(x, \lambda, v)\right] \frac{t^{n}}{n !}-m \sum_{n=0}^{\infty} n x^{-v_{m} m} v_{v}(x, \lambda, v) \frac{t^{n-1}}{n !} \\
=-m t \sum_{n=0}^{\infty} x^{-v} F_{m n+v}^{m}(x, \lambda, v) \frac{t^{n}}{n !}
\end{array}
$$


or

$$
\begin{aligned}
& \sum_{n=0}^{\infty}-v F_{m n+v}^{m}(x, \lambda, v) \frac{t^{n}}{n !}+x \sum_{n=0}^{\infty} D F_{m n+v}^{m}(x, \lambda, v) \frac{t^{n}}{n !} \\
& \quad-m \sum_{n=0}^{\infty} n F_{m n+v}^{m}(x, \lambda, v) \frac{t^{n}}{n !}=-m \sum_{n=1}^{\infty} F_{m(n-1)+v}^{m}(x, \lambda, v) \frac{t^{n}}{(n-1) !}
\end{aligned}
$$

Equating the coefficients of $t^{n}$ from both sides, for $n \geq 1$, we obtain the differential recurrence relation

$$
x \text { D F } F_{m n+v}^{m}(x, \lambda, v)-(\nu+m n) F_{m n+v}^{m}(x, \lambda, v)+m n F_{m(n-1)+v}^{m}(x, \lambda, v)=0 .
$$

To find out the other differential recurrence relation, let us start from the generating relation given by $(6.4)$, which can be written as:

$$
\sum_{n=0}^{\infty}\left(\frac{2 v+\lambda+m-1}{m}\right)_{n} x^{-v} F_{m n+v}^{m}(x, \lambda, v) \frac{t^{n}}{n !}=(1-t)^{-\frac{2 v+\lambda+m-1}{m}} \exp \left(\frac{-x^{m} t}{1-t}\right) .
$$

Let

$$
G(x, t)=(1-t)^{-\frac{2 v+\lambda+m-1}{m}} \exp \left(\frac{-x^{m} t}{1-t}\right)
$$

Writing $G$ in place of $G(x, t)$, and differentiating (7.9) partially with respect to $x$ :

$$
\frac{\partial G}{\partial x}=\frac{-m x^{m-1} t}{(1-t)} G \text {. }
$$

Substituting G from (7.8), we obtain

$$
\begin{aligned}
\sum_{n=0}^{\infty}\left(\frac{2 v+\lambda+m-1}{m}\right)_{n}\left[-v x^{-v-1} F_{m n+v}^{m}(x, \lambda, v)+x^{-v} D F_{m n+v}^{m}(x, \lambda, v)\right] \frac{t^{n}}{n !} \\
=\frac{-m x^{m-1} t}{(1-t)} \sum_{n=0}^{\infty}\left(\frac{2 v+\lambda+m-1}{m}\right)_{n} x^{-v} F_{m n+v}^{m}(x, \lambda, v) \frac{t^{n}}{n !}
\end{aligned}
$$

or

$$
\begin{aligned}
& \sum_{n=0}^{\infty}\left(\frac{2 v+\lambda+m-1}{m}\right)_{n}\left[-\frac{v}{x} F_{m n+v}^{m}(x, \lambda, v)+D F_{m n+v}^{m}(x, \lambda, v)\right] \frac{t^{n}}{n !} \\
& -\sum_{n=1}^{\infty}\left(\frac{2 v+\lambda+m-1}{m}\right)_{n-1}\left[-\frac{v}{x} F_{m(n-1)+v}^{m}(x, \lambda, v)+D F_{m(n-1)+v}^{m}(x, \lambda, v)\right] \frac{t^{n}}{(n-1) !} \\
& \quad=-m x^{m-1} \sum_{n=1}^{\infty}\left(\frac{2 v+\lambda+m-1}{m}\right)_{n-1} F_{m(n-1)+v}^{m}(x, \lambda, v) \frac{t^{n}}{(n-1) !} \cdot
\end{aligned}
$$

Equating the coefficients of $t^{n}$ from both sides, we obtain the differential recurrence relation 


$$
\begin{aligned}
& \left(\mathrm{n}+\frac{2 v+\lambda-1}{\mathrm{~m}}\right) \mathrm{F}_{\mathrm{m}+v}^{\mathrm{m}}(\mathrm{x}, \lambda, v)-\mathrm{x}\left(\mathrm{n}+\frac{2 v+\lambda-1}{\mathrm{~m}}\right) \mathrm{DF}_{\mathrm{mn}+v^{\mathrm{m}}}(\mathrm{x}, \lambda, \nu) \\
& -n\left(v+m x^{m}\right) F_{m(n-1)+v}^{m}(x, \lambda, v)+x n D F_{m(n-1)+v}^{m}(x, \lambda, v)=0 .
\end{aligned}
$$

7.2. PURE RECURRENCE RELATION. From the differential recurrence relation (7.7), we obtain

$$
x D F_{m n+v}^{m}(x, \lambda, v)=(\nu+m n) F_{m+v}^{m}(x, \lambda, v)-m n F_{m(n-1)+v}^{m}(x, \lambda, v)
$$

which implies that

$$
x \text { D F } F_{m(n-1)+v}^{m}(x, \lambda, v)=[v+m(n-1)] F_{m(n-1)+v}^{m}(x, \lambda, v)-m(n-1) F_{m(n-2)+v}^{m}(x, \lambda, v)
$$

To obtain the pure recurrence relation, we eliminate the derivatives from (7.11), (7.12) and (7.13), which yields

$$
\begin{aligned}
& \left(n+\frac{2 v+\lambda-1}{m}\right) F_{m+v}^{m}(x, \lambda, v)-\left(n+\frac{2 v+\lambda-1}{m}\right)\left[(v m n) F_{m n+v}^{m}(x, \lambda, v)-m n F_{m(n-1)+v}^{m}(x, \lambda, \nu)\right] \\
& -n\left(v+m x^{m}\right) F_{m(n-1)+v}^{m}(x, \lambda, v)+n[v+m(n-1)] F_{m(n-1)+v}^{m}(x, \lambda, v) \\
& -m n(n-1) F_{m(n-2)+v}^{m}(x, \lambda, v)=0 \text {. }
\end{aligned}
$$

Hence we obtain

$$
\begin{aligned}
\left(\mathrm{n}+\frac{2 v+\lambda-1}{\mathrm{~m}}\right) & \mathrm{F}_{\mathrm{m}+v}^{\mathrm{m}}(\mathrm{x}, \lambda, v)-\left[2 \mathrm{n}-1+\frac{2 v+\lambda-1}{\mathrm{~m}}-\mathrm{x}^{\mathrm{m}}\right] \\
& \times \mathrm{F}_{\mathrm{m}(\mathrm{n}-1)+v}^{\mathrm{m}}(\mathrm{x}, \lambda, \nu)+(\mathrm{n}-1) \mathrm{F}_{\mathrm{m}(\mathrm{n}-2)+v^{m}}(\mathrm{x}, \lambda, v)=0 .
\end{aligned}
$$

In the above equation we see that for a fixed $m$ and for different values of $V(\nu=0,1,2, \ldots, m-1)$, we shall obtain $m$ different recurrence relations. Also we are getting different recurrence relations for different partitions for the set of values of the variable parameter $\mathrm{n}$. For example if we take $\mathrm{m}=2$, then for $\nu=0(7.14)$ gives us the recurrence relation for even polynomials and for $\nu=1$ it gives us that for odd polynomials, which is interesting and seems to be new.

8. SPECIAL CASES.

CASE 1. If we put $m=1, \nu=0$ and $\lambda=1+\alpha$, then the differential equation (1.1) reduces to that for Laguerre polynomials as:

$$
x y^{\prime \prime}+(1+\alpha-x) y^{\prime}+n y=0 \text {. }
$$

By the same substitution, relation $(4.2)$ reduces to

$$
\mathrm{F}_{\mathrm{n}}^{1}(\mathrm{x}, 1+\alpha, 0)=\frac{\mathrm{n} !}{(1+\alpha)_{n}} \mathrm{~L}_{\mathrm{n}}^{(\alpha)} \quad(\mathrm{x}) \text {, }
$$


where $L_{n}^{(\alpha)}(x)$ is the Laguerre polynomials.

But for $\lambda=1$,

$$
F_{n}^{1}(x, 1,0)=L_{n}^{0}(x)=L_{n}(x),
$$

i.e. simple Laguerre polynomials.

Also for $\mathrm{m}=1, \nu=0$, and using the relation (8.1), the Rodrigues' formula (5.1), the generating functions given by (6.2) and (6.4) and recurrence relations (7.7), (7.11) and (7.14) reduce to that for Laguerre polynomials. For $\lambda=1$, these results reduce to that for simple Laguerre polynomials.

CASE 2. On putting $\mathrm{m}=2, \lambda=\nu=0$ differential equation (1.1) becomes

$$
y^{\prime \prime}-2 x y^{\prime}+2 n y=0,
$$

which is the differential equation for Hermite polynomials.

By the same substitution, relation (3.1) gives us,

$$
\begin{aligned}
F_{n}^{2}(x, 0,0) & =\frac{(-1)^{n / 2}}{\left(\frac{1}{2}\right)_{n / 2}} x^{n}{ }_{2} F_{0}\left(-\frac{n}{2},-\frac{n}{2}+\frac{1}{2} ;-;-\frac{1}{x^{2}}\right) \\
& =\frac{(-1)^{n / 2}}{\left(\frac{1}{2}\right)_{n / 2} 2^{n}} H_{n}(x),
\end{aligned}
$$

where $H_{n}(x)$ is the Hermite polynomial (see p. 108 of Rainville [8]). Once again, if we put $\mathrm{m}=2, \lambda=0, \nu=0$, in (4.1) then,

$$
\mathrm{F}_{2 \mathrm{n}}^{2}(\mathrm{x}, 0,0)={ }_{1} \mathrm{~F}_{1}\left(-\mathrm{n} ; \frac{1}{2} ; \mathrm{x}^{2}\right)=\frac{(-1)^{\mathrm{n}} \mathrm{n} !}{(2 \mathrm{n}) !} \mathrm{H}_{2 \mathrm{n}}(\mathrm{x}) ;
$$

and for $\mathrm{m}=2, \lambda=0, \nu=1$,

$$
\mathrm{F}_{2 \mathrm{n}+1}^{2}(\mathrm{x}, 0,1)=\mathrm{x}_{1} \mathrm{~F}_{1}\left(-\mathrm{n} ; \frac{3}{2} ; \mathrm{x}^{2}\right)=\frac{(-1)^{\mathrm{n}} \mathrm{n} !}{2(2 \mathrm{n}+1) !} \mathrm{H}_{2 \mathrm{n}+1}(\mathrm{x}) \text {; }
$$

where $\mathrm{H}_{2 \mathrm{n}}(\mathrm{x}), \mathrm{H}_{2 \mathrm{n}+1}(\mathrm{x})$ are even and odd Hermite polynomials, see Erdelyi [1], p. 194 .

With the help of these relations, we can calculate generating functions,

recurrence relations, etc. for the polynomials $H_{2 n}(x)$ and $H_{2 n+1}(x)$.

Next, when we put $\mathrm{m}=2, \nu=0$ in $(4.2)$, it reduces to generalized Hermite polynomials of Dutta, Chatterjea and Ghosh [3].

$$
\mathrm{F}_{2 \mathrm{n}}^{2}(\mathrm{x}, \lambda, 0)=\frac{\mathrm{n} !}{\left(\frac{\lambda+1}{2}\right)_{\mathrm{n}}} \mathrm{L}_{\mathrm{n}}^{\left(\frac{\lambda-1}{2}\right)}\left(\mathrm{x}^{2}\right)
$$




$$
=\frac{(-1)^{\mathrm{n}}}{\left(\frac{\lambda+1}{2}\right)_{n}} \mathrm{H}_{2 \mathrm{n}}(\mathrm{x})
$$

and for $m=2, \nu=1$, it reduces to:

$$
\begin{aligned}
\mathrm{F}_{2 n+1}^{2}(\mathrm{x}, \lambda, 1) & =\frac{x^{n} !}{\left(\frac{\lambda+3}{2}\right)_{n}} \mathrm{~L}_{\mathrm{n}}\left(\frac{\lambda+1}{2}\right)\left(x^{2}\right) \\
& =\frac{(-1)^{\mathrm{n}}}{\left(\frac{\lambda+3}{2}\right)_{n}} \mathrm{H}_{2 \mathrm{n}+1}^{\lambda}(\mathrm{x})
\end{aligned}
$$

where $H_{2 n}^{\lambda}(x)$ and $H_{2 n+1}^{\lambda}(x)$ are defined in Dutta, Chatterjea and Ghosh [3].

\section{REFERENCES}

1. ERDELYI, A. et a1. Higher Transcendental Functions, Vo1. II, McGraw-Hill, New York, Toronto and London, 1953.

2. DUTTA, M. and MORE, K.L. A New Class of Generalized Legendre Polynomials, Mathematica 7 30 (1965) 33-41.

3. DUTTA, M., CHATTERJEA, S.K. and GHOSH, B.K. On Generalized Hermite Polynomials, Bull. Math. Soc. Sci. Math. R.S. de Roumanie 12 60, No. 4 (1968) 59-65.

4. DUTTA, M., CHATTERJEA, S.K. and MORE, K.L. On a class of Generalized Hermite Polynomials, Bul1. Inst. of Math., Academia Sinica. 3, No. 2 (Dec. 1975 ).

5. KRALL, H.L. and FRINK, 0. A New Class of Orthogonal Polynomials: The Besse1 Polynomia1s, Trans. Amer. Math. Soc. 65 (1949) 100-115.

6. DUTTA, M. and BHATTACHARYA, S.P. The Function Space Properly Associated with the Class of Differential Equations, Bul1. Cal. Math. Soc. 62 (1970) 183-188.

7. DUTTA, M. Orthogonality and Differential Equations, Bul1. Cal. Math. Soc. 63 (1971), 143-145.

8. RAINVILLE, E.D. Special Functions, MacMillan Company, New York, 1960. 


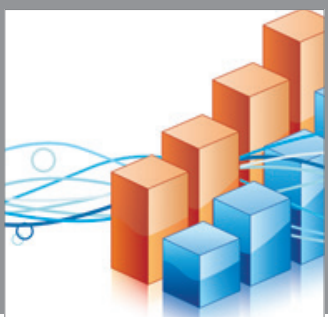

Advances in

Operations Research

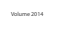

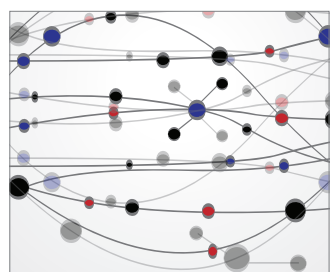

\section{The Scientific} World Journal
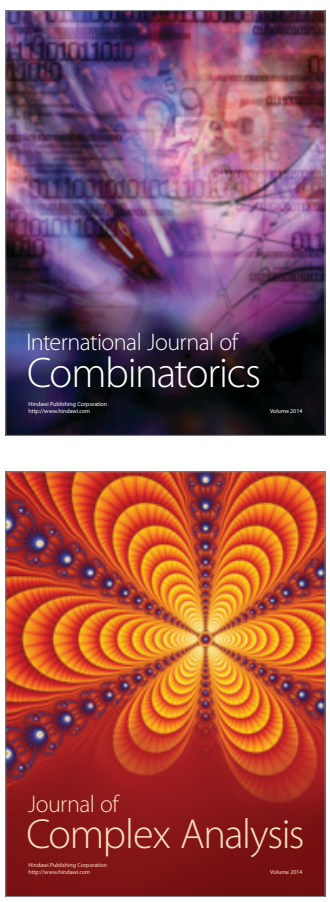

International Journal of

Mathematics and

Mathematical

Sciences
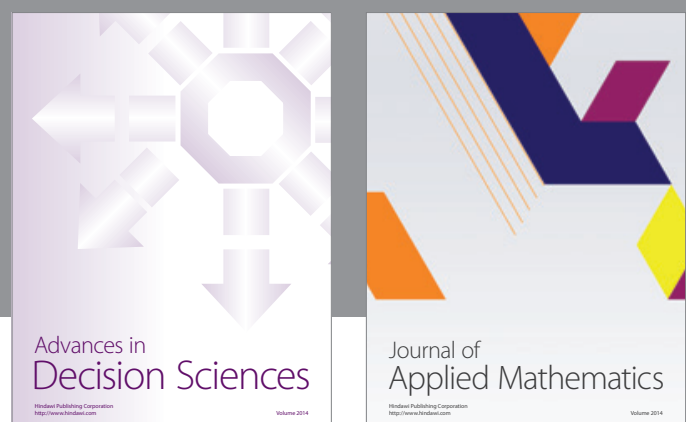

Journal of

Applied Mathematics
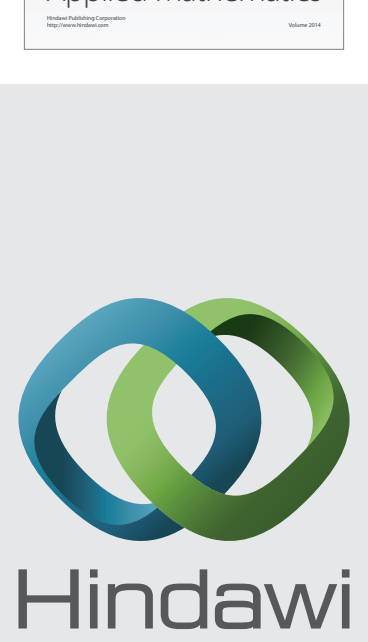

Submit your manuscripts at http://www.hindawi.com
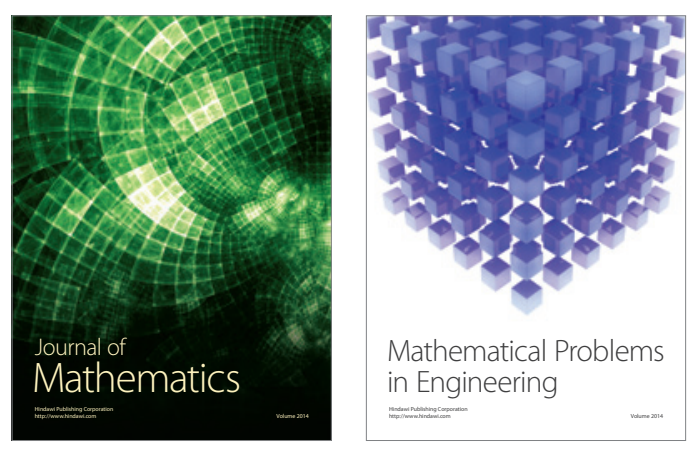

Mathematical Problems in Engineering
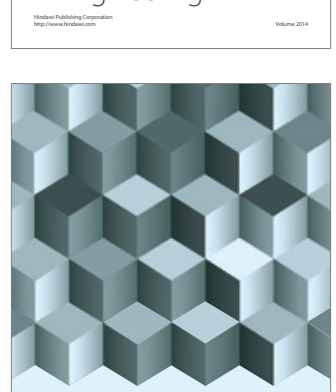

Journal of

Function Spaces
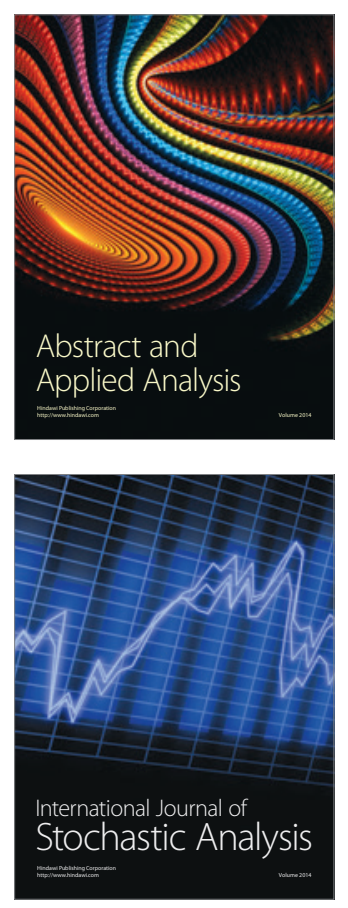

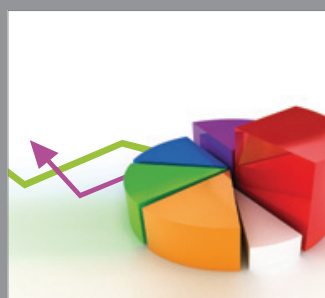

ournal of

Probability and Statistics

Promensencen
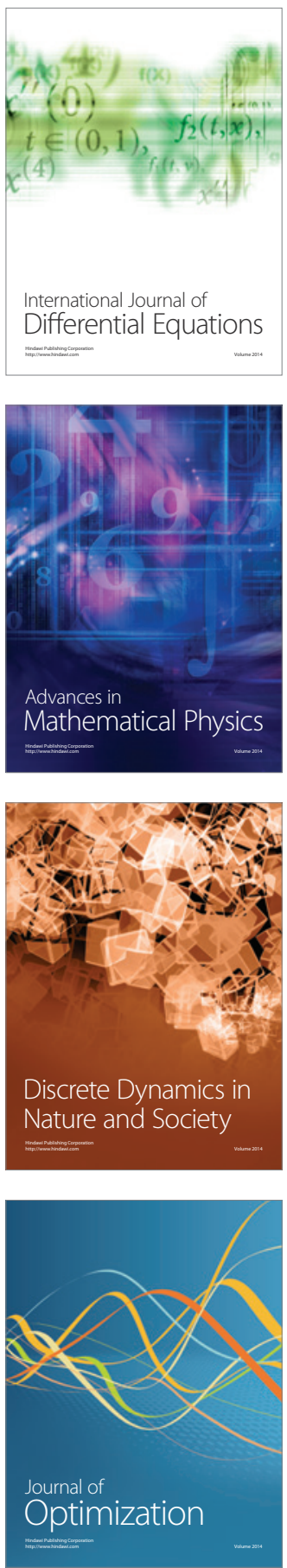